\title{
Uso de computador e ergonomia: um estudo sobre as escolas de ensino fundamental e médio de São Paulo
}

Lys Esther Rocha

Raquel Aparecida Casarotto

Laerte Sznelwar

Universidade de São Paulo

\begin{abstract}
Resumo
A utilização da informática como instrumento de ensino tem se disseminado na educação. Este estudo teve como objetivo verificar como o computador está sendo usado em escolas da região metropolitana de São Paulo, avaliando o grau de considerações sobre ergonomia na introdução dessa ferramenta. A ergonomia compreende o conjunto de conhecimentos científicos visando o conforto, segurança e eficácia dos produtos.

Participaram 126 escolas, 37 delas do ensino fundamental, de $1^{\text {a }}$ à 4 a série, com 21.824 alunos; 49 escolas do ensino fundamental, de 5 a à 8 a série, com 29.851 alunos e 40 escolas do ensino médio com 31.516 alunos. A maioria das escolas informou utilizar o computador como ferramenta auxiliar das matérias curriculares a partir de 1995. 0 uso do computador predominou, no período de 5 à à 8 $^{a}$ série, nas escolas particulares; com um computador para cada 2 ou 3 alunos e com a duração semanal das aulas de até 1 h59min. Nas salas de informática instaladas nas escolas, predominou a ausência de mesas e cadeiras com ajustes de altura para as características antropométricas dos alunos. As orientações sobre ergonomia da computação foram fornecidas em $30 \%$ das escolas. Na maioria das escolas não se observou queixa de desconforto visual ou muscular sofridos pelos alunos durante a utilização do computador. Esse fato pode estar relacionado ao pequeno número de horas de utilização do computador.

Concluímos que a aquisição de mobiliário e equipamentos para as salas de informática deve incluir a consulta a banco de dados antropométricos e à percepção de conforto dos estudantes.
\end{abstract}

\author{
Palavras-chave \\ Informática - Escolas - Computador - Ergonomia.
}




\title{
Ergonomics and the use of computers: a study with primary and secondary schools in the State of São Paulo
}

\author{
Lys Esther Rocha \\ Raquel Aparecida Casarotto \\ Laerte Sznelwar \\ Universidade de São Paulo
}

\begin{abstract}
The use of computers as a teaching tool is becoming disseminated in education. The present study has as its purpose to investigate how computers are being used in schools within the metropolitan area of São Paulo, evaluating the level of ergonomic issues considered when introducing this tool. Ergonomics comprise the collection of scientific knowledge to ensure the comfort, safety, and efficacy of products.

The work included 126 schools distributed as follows: 37 primary schools (1st to 4th year) with 21,824 students; 49 primary schools (5th to 8th year) with 29,851 students; and 40 secondary schools with 31,516 students. Most schools have declared to use computers as an auxiliary tool in their syllabuses since 1995. The use of computers in the 5th to 8th year of schooling was predominant in private schools, with an average of two to three students per computer, and an average duration of classes of up to 1h59min per week. The majority of tables and chairs installed in computer classrooms at schools lack height adjustments to fit the anthropometric characteristics of pupils. Thirty percent of schools supplied computer ergonomics guidelines. In most of the schools no complaints were observed regarding students' visual or muscular discomfort during the use of computers. This fact can be related to the small number of hours of use.

We have concluded that the acquisition of furniture and equipment for computer classrooms should include consulting anthropometric databases and students' perception of comfort.
\end{abstract}

\section{Keywords}

Computing - Schools - Computers - Ergonomics.

Contact:

Lys Esther Rocha

Rua Teodoro Sampaio, 115

05405-000 - São Paulo/SP

e-mail: lysrocha@usp.br 
A utilização de computadores na educação tem trazido diferentes tipos de discussão. La Faille (1989), Crochik (1990) e Bittencourt (1998) analisam a problemática da informática na educação do ponto de vista epistemológico e didático, caracterizando o computador como um recurso didáticopedagógico. Uma das preocupações desses pesquisadores reside no fato de que o computador estimularia a operacionalização de conceitos, empobrecendo o raciocínio dos estudantes. Por outro lado, o desenvolvimento de softwares com linguagem de programação, como Basic, Pascal e Logo, permitiram ao aluno vivenciar o seu potencial criativo, uma vez que possibilitam ao estudante utilizar o computador como máquina a ser ensinada, podendo resolver problemas, finalizar tarefas, desenhar, escrever (Valente, 2002; Prado, 2002).

Entre as vantagens da utilização das tecnologias de informática nas escolas, Knave (1997) cita o desenvolvimento da arte de educar e treinar que, com o uso das tecnologias, torna mais agradável a aula para os estudantes, fazendo da escola um lugar mais agradável, excitante e variável, tornando os alunos mais motivados e concentrados, e possibilitando que os estudantes obtenham respostas rápidas, desenvolvam a cooperação e a solidariedade. $\mathrm{Na}$ Suécia, em 1994, foi desenvolvida uma política para o uso da tecnologia da informática na educação enfatizando que os alunos devem aprender a buscar informações e se comunicar por computadores pessoais, a utilizar programas de processadores de texto e cálculo e que os professores devem receber treinamento nesse tipo de tecnologia.

Além dos aspectos pedagógicos, o uso do computador na escola suscita discussões sobre a utilização da ergonomia no planejamento do ambiente escolar e na definição de orientações preventivas aos professores e estudantes. Entendendo-se como ergonomia "0 conjunto de conhecimentos científicos relativos ao homem e necessários para a concepção de ferramentas, máquinas e dispositivos que possam ser utilizados com o máximo de conforto, segurança e eficácia" (Wisner, 1987, p.12).

Bartolomeu et al. (1999) analisam o dimensionamento correto do mobiliário dos laboratórios de informática das escolas, discutindo desde a distribuição do mobiliário nas salas de informática até as características das mesas, cadeiras e microcomputadores.

Levando-se em conta que, no momento atual, o emprego da informática na educação tem se disseminado nas escolas em São Paulo, este estudo teve por objetivo verificar como 0 computador está sendo utilizado, avaliando o grau de considerações sobre ergonomia na introdução dessa ferramenta, no planejamento do local de sua utilização e nas orientações fornecidas aos professores e alunos.

\section{Metodologia}

Este estudo envolveu a participação de 125 escolas da região metropolitana de São Paulo, divididas em:

a) ensino fundamental de 1 a à 4 a série, 37 escolas, 21.824 alunos;

b) ensino fundamental de $5^{\text {a }}$ à $8^{\underline{a}}$ série, 49 escolas, 29.851 alunos;

c) ensino médio, 40 escolas, 31.516 alunos.

As escolas foram selecionadas buscando abranger diferentes localizações geográficas da região metropolitana de São Paulo, como também a natureza pública ou privada da instituição. Não houve a intenção de fornecer um quadro representativo da utilização de computadores pelas escolas em São Paulo, mas o objetivo de compreender como o computador tem sido usado no ensino fundamental e médio.

0 questionário aplicado foi elaborado originalmente em inglês, por Bergqvist et al. (1997), do National Institute for Working Life, em Estocolmo, na Suécia, com o objetivo de realizar uma pesquisa internacional sobre a utilização de computadores nas escolas. 0 
questionário foi utilizado na Suécia e depois traduzido e aplicado no J apão, Itália, Noruega, Chile, Austrália, Finlândia e Estados Unidos.

A primeira etapa do presente estudo consistiu na tradução do questionário do inglês para o português por docentes da Faculdade de Medicina e da Escola Politécnica da Universidade de São Paulo. A tradução foi testada em cinco escolas do ensino fundamental e médio para avaliar a compreensão do seu conteúdo. 0 conteúdo do questionário caracteriza a existência de cursos de informática ou a utilização de computadores como auxiliar nas demais matérias; o tempo de utilização dos computadores pelos alunos; as características das salas de informática e equipamentos; as orientações fornecidas na utilização dos computadores e as queixas de desconforto referidas pelos estudantes.

A segunda etapa consistiu no treinamento de alunos do curso de Fisioterapia da Universidade de São Paulo, que estavam participando da disciplina Fisioterapia Preventiva. Foi lido o questionário e discutido o conteúdo de cada questão.

A coleta de dados nas escolas foi feita no período entre maio e setembro de 1998, pelos alunos do curso de Fisioterapia, sob a supervisão de docentes da Faculdade de Medicina e Escola Politécnica da Universidade de São Paulo. Nas escolas, as entrevistas foram realizadas com os professores de informática; não havendo esse cargo, as questões foram dirigidas à direção da escola. Em cada escola, as respostas foram preenchidas para apenas um período escolar (ensino fundamental de $1^{\text {a }}$ à $4^{\text {a }}$ série ou de $5^{\text {a }}$ à $8^{\text {a }}$ série ou do ensino médio).

Os dados dos questionários foram codificados e digitados com análise de consistência das informações. Neste estudo realizamos a análise descritiva dos resultados.

\section{Resultados}

0 número médio de alunos das escolas em cada estágio de ensino foi de 590 no ensino fundamental de $1^{a}$ à $4^{a}$ série, correspondendo a um computador para cada 24 alunos; 609 alunos de 5 a à 8 a série, com um computador para cada 27 alu- nos; e 788 alunos no ensino médio, com um computador para cada 22 alunos. A maioria das escolas informou utilizar o computador a partir de 1995.

A utilização do computador variou de acordo com o ano escolar, com índice de $62,2 \%$ para o ensino fundamental de 1 a à 4 a série, de $77,6 \%$ para 0 ensino fundamental de 5 a à 8 a série e de $62,5 \%$ para 0 ensino médio. Quanto à natureza pública ou privada das escolas, as escolas públicas apresentaram menor uso do computador em todos os anos de ensino (Tabela 1).

Tabela 1. Dimensão do uso do computador pelas escolas de São Paulo segundo o nível de escolaridade, a natureza da instituição e o tipo de utilização.

\begin{tabular}{|c|c|c|c|c|c|c|}
\hline \multirow{2}{*}{$\begin{array}{l}\text { Uso do Computador } \\
\text { Frequiência de uso }\end{array}$} & \multicolumn{2}{|c|}{$\begin{array}{l}\text { Fundamental } \\
1^{\mathrm{a}} \text { à } 4^{\mathrm{a}} \text { série } \\
(\mathrm{N}=37)\end{array}$} & \multicolumn{2}{|c|}{$\begin{array}{l}\text { Fundamental } \\
5^{\mathrm{a}} \text { à } 8^{\mathrm{a}} \text { série } \\
(\mathrm{N}=49)\end{array}$} & \multicolumn{2}{|c|}{$\begin{array}{l}\text { Médio } \\
(\mathrm{N}=40)\end{array}$} \\
\hline & $\mathrm{N}$ & $\%$ & $\mathrm{~N}$ & $\%$ & $\mathrm{~N}$ & $\%$ \\
\hline Conjunto das escolas & 23 & $(62,2)$ & 38 & $(77,6)$ & 25 & $(62,5)$ \\
\hline Natureza da escola & $\mathrm{N}$ & $\%$ & $\mathrm{~N}$ & $\%$ & $\mathrm{~N}$ & $\%$ \\
\hline Pública* & 8 & $(53,3)$ & 10 & $(52,6)$ & 3 & $(33,3)$ \\
\hline Privada** & 15 & $(68,2)$ & 28 & $(93,3)$ & 22 & $(71,0)$ \\
\hline Cursos de informática & $\mathrm{N}$ & $\%$ & $\mathrm{~N}$ & $\%$ & $\mathrm{~N}$ & $\%$ \\
\hline Não oferecido & 17 & $(45,9)$ & 18 & $(36,7)$ & 17 & $(42,5)$ \\
\hline 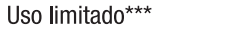 & 18 & $(48,6)$ & 18 & $(36,7)$ & 15 & $(37,5)$ \\
\hline Uso extensivo & 2 & $(5,4)$ & 13 & $(26,5)$ & 8 & $(20,0)$ \\
\hline
\end{tabular}

Computador no

$\begin{array}{lclllll}\text { auxílio de matérias } & \mathrm{N} & \% & \mathrm{~N} & \% & \mathrm{~N} & \% \\ \text { Não oferecido } & 14 & (37,8) & 14 & (28,6) & 16 & (40,0) \\ \text { Uso limitado } & 4 & (10,8) & 16 & (32,6) & 10 & (25,0) \\ \text { Uso extensivo } & 19 & (51,4) & 19 & (40,0) & 14 & (35,0)\end{array}$

* $\quad$ total de escolas públicas de 1 a à 4 a série foi de 15 escolas, de 5a à 8 a série foi de 19 escolas e no ensino médio foi de 9 escolas.

** 0 total de escolas privadas de 1 a à 4 a série foi de 22 escolas, de 5 a à 8 a série foi de 30 escolas e no ensino médio foi de 31 escolas.

*** o uso limitado ou extensivo do computador para cursos de informática ou no auxílio de matérias corresponde à abrangência para o conjunto dos alunos em cada série. Paulo segundo o nível de escolaridade

Em relação ao emprego extensivo do computador, a maioria das escolas, em todos os níveis de ensino, utilizou o computador principalmente como ferramenta auxiliar das matérias curriculares e em menor proporção para cursos de informática. Para os alunos da 1a à 4 a série 
do ensino fundamental, essa diferença foi mais acentuada: $51,4 \%$ das escolas usam o computador como ferramenta no auxílio de outras disciplinas e 5,4\% oferecem cursos específicos de informática. Por outro lado, no ensino mé-

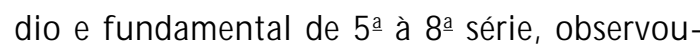
se maior oferta de cursos de informática para os alunos (Tabela 1).

Em relação aos cursos de informática (Tabela 2), observou-se o predomínio da oferta de cursos de processadores de texto e criação de imagens em todos os níveis de ensino. Em relação a cursos que habilitassem à utilização da Internet, houve predomínio da oferta para os alunos do curso médio.

Tabela 2. Tipos de cursos de informática oferecidos pelas escolas de São Paulo segundo o nível de escolaridade

$\begin{array}{lcclllll}\begin{array}{l}\text { Tipo de Cursos } \\ \text { de Informática }\end{array} & \begin{array}{l}\text { Fundamental } \\ 1^{\mathrm{a}} \text { à } 4^{\mathrm{a}} \text { série } \\ (\mathrm{N}=23)\end{array} & \begin{array}{l}\text { Fundamental } \\ 5^{\mathrm{a}} \text { à } 8^{\mathrm{a}} \text { série } \\ (\mathrm{N}=38)\end{array} & \begin{array}{l}\text { Médio } \\ (\mathrm{N}=25)\end{array} \\ \begin{array}{l}\text { Freqüência de } \\ \text { oferecimento }\end{array} & \mathrm{N} & \% & \mathrm{~N} & \% & \mathrm{~N} & \% \\ \text { Processador de texto } & 14 & (60,9) & 29 & (76,3) & 18 & (72,0) \\ \text { Criação de imagens } & 17 & (73,9) & 19 & (50,0) & 12 & (48,0) \\ \text { Internet } & 6 & (26,1) & 13 & (34,2) & 11 & (44,0) \\ \text { Programação } & 7 & (30,4) & 5 & (13,2) & 6 & (24,0) \\ \text { Outros } & 11 & (47,8) & 17 & (44,7) & 10 & (40,0)\end{array}$

Em relação ao modo de utilização do computador (Tabela 3), verificou-se o predomínio do uso de um computador para cada dois a três alunos, com a duração semanal das aulas de até 1 h59min em todos os estágios. Com relação à disponibilidade de utilização do computador, esta se deu em $72 \%$ das escolas para os alunos do ensino médio e em apenas 34\% delas para os alunos do ensino fundamental, 0 que demonstra um uso diferenciado de acordo com o estágio do aluno.

Observou-se, nas escolas, a construção ou adaptação de salas para o uso dos computadores, conhecidas como Salas de Informática (Tabela 4). Esses locais são utilizados por alunos
Tabela 3. Aspectos da utilização do computador pelas escolas de São Paulo segundo o nível de escolaridade

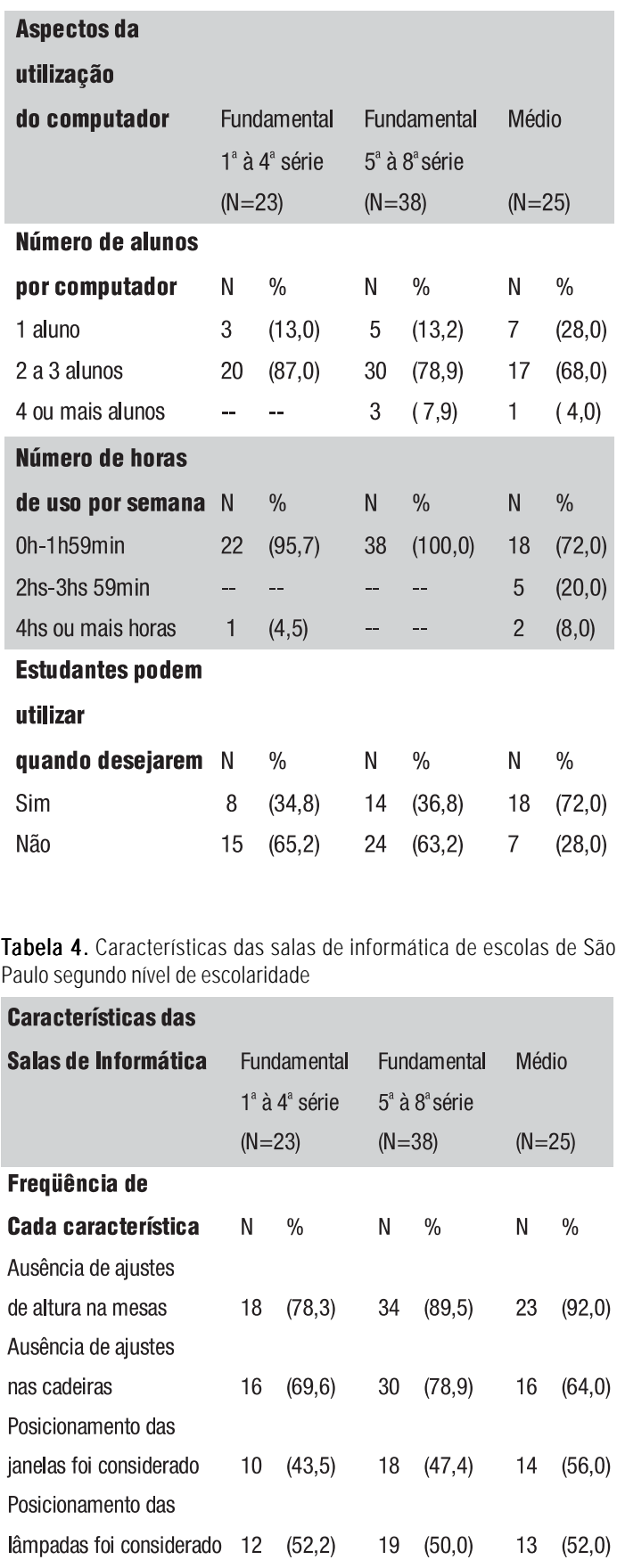

do ensino fundamental e médio, predominando a ausência de mesas e cadeiras com ajustes de altura, isto é, sem adaptação do mobiliário para 
as diferentes estaturas entre alunos de 7 a 17 anos. Em torno de $50 \%$ das escolas, foi planejado o posicionamento das janelas e lâmpadas, durante a instalação dos computadores, visando a evitar a presença de reflexos nos terminais.

Em relação às características dos computadores utilizados, todas as escolas utilizavam 0 computador tipo Desktop, com telas CRT e coIoridas, com uso do teclado e mouse para a entrada de dados.

Em relação às orientações fornecidas para os alunos quanto ao modo de uso dos computadores (Tabela 5), em torno de $30 \%$ das

Tabela 5. Orientações aos alunos sobre o uso dos computadores em escolas de São Paulo segundo nível de escolaridade

$\begin{array}{lllllll}\begin{array}{l}\text { Conteúdo das } \\ \text { Orientações }\end{array} & \begin{array}{l}\text { Fundamental } \\ 1^{\mathrm{a}} \text { à } 4^{\mathrm{a}} \text { série } \\ (\mathrm{N}=23)\end{array} & \begin{array}{l}\text { Fundamental } \\ 5^{\mathrm{a}} \text { à } 8^{\mathrm{a}} \text { série } \\ (\mathrm{N}=38)\end{array} & \begin{array}{l}\text { Médio } \\ (\mathrm{N}=25)\end{array} \\ \begin{array}{l}\text { Freqüência de } \\ \text { Cada característica }\end{array} & \mathrm{N} & \% & \mathrm{~N} & \% & \mathrm{~N} & \% \\ \begin{array}{l}\text { Ergonomia na } \\ \text { Computação }\end{array} & 8 & (34,7) & 10 & (26,4) & 7 & (28,0) \\ \begin{array}{l}\text { Postura na cadeira } \\ \text { (2) }\end{array} & 21 & (91,3) & 23 & (60,5) & 16 & (64,0)\end{array}$

Tabela 6. Queixas de desconforto dos alunos quando utilizam os computadores nas escolas de São Paulo segundo nível de escolaridade

\begin{tabular}{|c|c|c|c|c|c|c|}
\hline $\begin{array}{l}\text { Localização } \\
\text { das Queixas }\end{array}$ & \multicolumn{2}{|c|}{$\begin{array}{l}\text { Fundamental } \\
1^{\mathrm{a}} \text { à } 4^{\mathrm{a}} \text { série } \\
(\mathrm{N}=23)\end{array}$} & \multicolumn{2}{|c|}{$\begin{array}{l}\text { Fundamental } \\
5^{\mathrm{a}} \text { à } 8^{\mathrm{a}} \text { série } \\
(\mathrm{N}=38)\end{array}$} & \multicolumn{2}{|c|}{$\begin{array}{l}\text { Médio } \\
(\mathrm{N}=25)\end{array}$} \\
\hline \multicolumn{7}{|l|}{ Freqüência de } \\
\hline Cada característica & $\mathrm{N}$ & $\%$ & $\mathrm{~N}$ & $\%$ & $\mathrm{~N}$ & $\%$ \\
\hline Ausência de queixas & & & & & & \\
\hline de desconforto & 17 & $(73,9)$ & 30 & $(78,9)$ & 18 & $(72,0)$ \\
\hline \multicolumn{7}{|c|}{ Desconfortos relacionados } \\
\hline ao sistema ocular & 3 & $(13,0)$ & 3 & $(7,9)$ & 5 & $(20,0)$ \\
\hline \multicolumn{7}{|l|}{ Desconfortos relacionados } \\
\hline ao pescoço e ombros & -- & -- & -- & -- & 1 & $(4,0)$ \\
\hline \multicolumn{7}{|c|}{ Desconfortos relacionados } \\
\hline aos braços e mãos & -- & -- & -- & -- & 2 & $(8,0)$ \\
\hline \multicolumn{7}{|c|}{ Desconfortos relacionados } \\
\hline às costas & 2 & $(8,7)$ & 2 & $(5,3)$ & 3 & $(12,0)$ \\
\hline
\end{tabular}

escolas relataram oferecer algum curso sobre ergonomia na computação. As recomendações sobre a postura a ser adotada nas cadeiras foi fornecida para $91,3 \%$ dos alunos do curso fundamental da 1a à 4 a série e para $60 \%$ dos alunos dos outros estágios.

$\mathrm{Na}$ tabela 6 caracterizou-se a observação dos professores de informática em sobre a presença de queixas de desconforto pelos alunos durante a utilização dos computadores. $\mathrm{Na}$ maioria das escolas não foram observadas queixas. 0 desconforto mais citado foi o relacionado ao sistema ocular, que aconteceu em $20 \%$ dos alunos do ensino médio, $13 \%$ do ensino fundamental de 1a à 4a série e $7,9 \%$ para os alunos do ensino fundamental de $5^{a}$ à $8^{a}$ série. 0 desconforto relacionado às costas ocorreu em $12 \%$ do ensino médio.

\section{Disc us são}

Este estudo mostrou um crescente uso de computadores nas escolas da região metropolitana de São Paulo, principalmente nas instituições privadas, com o planejamento de salas de informática que estão sendo utilizadas por alunos dos diferentes estágios (ensino fundamental e médio) sem um adequado dimensionamento do mobiliário para a variabilidade de dimensões corporais dos estudantes.

Bergqvist et al. (1997) aplicaram o mesmo questionário, utilizado neste estudo, em Estocolmo, observando que o uso mais freqüente do computador aconteceu como ferramenta de ensino auxiliar de outras matérias. Nas escolas então pesquisadas havia dez estudantes para cada computador. No ensino médio a utilização individual do computador era comum. Foram organizadas salas de informática e a maioria utilizava computador desktop e colorido. A minoria das escolas havia fornecido orientações sobre ergonomia e postura. No momento da instalação dos computadores, foram considerados os posicionamentos das janelas e lâmpadas. Esses dados são similares aos encontrados nesta pesquisa, exceção feita ao fato de que o número 
de usuários por microcomputador no ensino médio em São Paulo é maior que 1(um).

No presente estudo verificou-se a utilização dos computadores por mais de uma criança. Esse fato também foi observado por Noro et al. (1997), em inquérito sobre o uso de computadores em escolas do ensino fundamental de $1^{\mathrm{a}}$ à $4^{\mathrm{a}}$ série, no J apão, onde um posto com computador é dividido por duas ou três crianças e o tamanho geral do mobiliário é grande quando comparado à estatura das crianças.

Nas escolas estudadas predominou 0 uso de computadores desktop com as queixas de desconforto nas costas variando de 5 a $12 \%$, enquanto Harris e Straker, (2000) ao analisar 0 uso de laptop por crianças de 10 a 17 anos, na Austrália, em casa e escola, verificaram que 20 a 38\% relataram desconforto na coluna lombar quando usavam ou carregavam o equipamento.

Neste estudo verificou-se um índice pequeno de queixas de desconforto para o sistema visual e coluna, resultado também observado no estudo de Bergqvist et al. (1997). Esse fato pode ser explicado pelo pouco tempo de uso dos computadores pelos alunos nas escolas.

0 dimensionamento correto do mobiliário para escolas é uma preocupação internacional, tendo gerado uma padronização, que no Brasil foi definida pela Associação Brasileira de Normas Técnicas (ABNT) sob o número 14.006. Entretanto, esses parâmetros estão sendo discutidos por diversos autores (Mandal, 1982; Aagaard-Hansen et Storr-Paulsen, 1995; Troussier et al., 1999).

Noro et al. (1997) sugerem o planejamento específico para o mobiliário com a inclusão de ajustes nas mesas e cadeiras de acordo com as medidas dos alunos, acompanhada da colocação do monitor do computador diretamente na mesa, do fornecimento de suporte para os pés quando as crianças não alcançarem o chão e a preocupação com o apoio das mãos e braços dos alunos.

No Brasil, Bartolomeu et al. (1999) analisaram o mobiliário de informática destinado a crianças de 5 a 11 anos e apresentaram algumas orientações em relação à postura e características desses equipamentos:

- Cadeira: o assento e o encosto devem ser estofados e revestidos de material perspirante, com densidade e consistência para suportar até $2 \mathrm{~cm}$ de depressão. 0 assento e 0 encosto devem ser ajustáveis para a altura, permitindo que o estudante apoie totalmente os pés no chão e garantam o suporte lombar. 0 assento deve ter as bordas arredondadas. Os pés da cadeira devem ser estáveis, com cinco pés e rodízio para sua movimentação.

- Mesa: deve permitir que o aluno fique posicionado com os ombros relaxados e o ângulo entre o braço e 0 antebraço seja de 90 graus. A mesa deve ser feita de material anti-reflexivo.

- Apoio para os pés: deve apresentar uma inclinação de 20 graus, para melhorar a postura e o retorno venoso do usuário.

- Posicionamento dos equipamentos: 0 terminal de vídeo deve ficar a 90 graus do plano da mesa, sendo sua distância para os olhos de 35 a $50 \mathrm{~cm}$. A altura do terminal de vídeo deve ser de dez graus abaixo da linha do horizonte visual do usuário.

A partir do artigo de Morgado et al. (2002), sobre recomendações para criação de ambientes informatizados em escolas públicas, evidenciam-se as divergências existentes sobre altura de mesa e cadeiras, uma vez que esse autor recomenda alturas fixas para os dois equipamentos, enquanto Bartolomeu et al. (1999) propõem regulagem para ambos.

A aquisição de mobiliário e equipamentos para o laboratório de informática deve incluir a consulta a banco de dados antropométricos e também a percepção de conforto dos estudantes na utilização desses instrumentos, conforme proposto por Aagard-Hansen e Storr-Paulsen (1995). Casarotto e Liberti (1994) desenvolveram bancos de dados com medidas dos segmentos corporais de escolares brasileiros na faixa etária de 4 a 7 anos e Silva e Paschoarelli (1999) na faixa de 3 a 18 anos.

No planejamento da sala de informática ressalta-se que, em relação à iluminação, as 
principais tarefas colocam duas exigências quase opostas.

A leitura do texto e o olhar para o teclado requerem um nível de iluminação elevado, enquanto a leitura da informação na tela do monitor, exige um bom contraste entre os caracteres e 0 fundo. Pela sua natureza este contraste diminui em função do aumento do nível de iluminação do local por interferência da luz. (Lips et al., 1991, p. 26)

É preciso colocar as luminárias de maneira a evitar os reflexos na superfície da tela. Algumas recomendações incluem a necessidade de não haver janelas em frente ou atrás do monitor; e de o ângulo principal do olhar ser paralelo à fila de janelas e de luminárias.

Neste estudo observou-se uma preocupação das escolas em orientar sobre a postura correta na cadeira. Contudo o dimensionamento inadequado do mobiliário e a falta de possibilidade de ajustá-los torna ineficazes essas orientações para os alunos, para os programas de prevenção de dores na coluna e lesões por esforços repetitivos na população trabalhadora.
Mattos (1990), ao analisar o processo de formação de professores de segundo grau em informática educacional, mostra a ausência desse conteúdo nos programas desenvolvidos. Nas propostas de Almeida (2002), para um curso de especialização em informática em educação, esses conteúdos também estão ausentes.

\section{C onc lusã o}

Este estudo verificou que o computador é uma ferramenta de ensino consolidada nas escolas. Apesar disso, a adequação das salas de informática às características antropométricas dos estudantes ainda não foi implementada. Recomenda-se que, na aquisição de mobiliários novos, ou reestruturação dos ambientes, as orientações sobre ergonomia sejam incorporadas.

Os professores de informática deveriam também receber noções de ergonomia, para que pudessem repassar esses conhecimentos nas aulas de informática, ajudando assim a criar nos estudantes uma postura preventiva com relação aos problemas que o uso do computador e os ambientes inadequados podem ocasionar nos seus desempenhos e na saúde.

\section{Referências bibliográfic as}

AAGAARD-HANSEN, I .; STOOR-PAULSEN, A. A comparative study of three different kinds of school furniture. Ergonomics, London, V. 38, n. 5, p. $1025-1035,1995$.

ALMEIDA, M. E. B. de. Informática e formação de professores, 2002. Disponível em: <http://www.proinfo.mec.gov.br> Acesso em: 14 maio 2002.

BARTOLOMEU, T. A.; TODESCO, R. P. R.; ABIB, S. W.; PETROSKI, E. L.; BASTOS, R. C. Avaliação do mobiliário de um laboratório de informática destinado às crianças de 5 a 11 anos sob uma visão antropométrica. In: Congresso Braslleiro de ERgonomia, 9., 1999, Salvador. Anais. Rio de J aneiro: ABERGO, 1999. Cd-rom.

BERGQVIST, U.; SOTOYAMA, M.; SAITO, S.; PICCOLI, B. Computers in schools: an international project under planning. In: INTERNATIONAL SCIENTIFIC CONFERENCE ON WORK WITH DISPLAY unITS, 5., 1997, Tóquio. Proceedings. Tóquio, 1997. p.213-214.

BITTENCOURT, J . Informática na educação? algumas considerações a partir de um exemplo. Revista da Faculdade de Educação, São Paulo, v. 24, n.1, jan/jun. 1998.

CASAROTTO, R. A.; LIBERTI, E. A. Dados antropométricos de pré-escolares da cidade de São Paulo. Revista de Fisioterapia da Universidade de São Paulo, São Paulo, v. 1, n. 1, p. 27- 33, jul/dez.1994. 
CROCHIK, J . L. 0 ajustamento do pensamento em uma sociedade de alto desenvolvimento tecnológico: o computador no ensino. São Paulo; 1990. 433p. Tese (Doutorado) - Instituto de Psicologia da Universidade de São Paulo.

HARRIS, C.; STRAKER, L. Survey of physical ergonomics issues associated with school children's use of laptop computers. International J ournal of Industrial Ergonomics, Amsterdã, v. 26, n. 3, p. 337- 346, 2000.

KNAVE, B. Information Technology (IT) in schools. In: INTERNATIONAL SCIENTIFIC CONFERENCE ON WORK WITH DISPLAY UNITS, 5., 1997, Tóquio. Proceedings. Tóquio, 1997. p.107- 108.

LA FAILLE, Y. J .J . M. R. de. Ensaio sobre o lugar do computador na educação: relato do Projeto Ciranda/São Paulo e o tema análise de resposta. São Paulo; 1989. 910p. Tese (Doutorado) - Instituto de Psicologia da Universidade de São Paulo.

LIPS, W.; WEICKHARDT, U.; BUCHBERGER, J .; KRUEGER, H. Le travail à I'écran de visualisation. Zurique: Caisse Nationale Suisse d'Assurance en Cas d'Accidents Sécurité au Travail, 1991.65 p.

MANDAL, A C. The correct height of school furniture. Human Factors, Nova York, v. 24, n. 3, p. 257- 269, 1982.

MATTOS, M. I. L. de. 0 computador na escola pública: análise do processo de formação de professores de segundo grau no uso desta tecnologia. 1990. 258p. Tese (Doutorado) - Instituto de Psicologia da Universidade de São Paulo, São Paulo, 1990.

MORGADO, E. M.; CAVENAGUI, M. A.; REINHARD, N. Preparação de ambientes informatizados nas escolas públicas, 2002. Disponível em: <http://www.proinfo.mec.gov.br> Acesso em: 14 maio 2002.

NORO, K.; OKAM OTO, T.; KOJ IMA, M. Computer operation by primary school children in J apan. In: InTernational Scientific Conference On Work With Display Units, 5., 1997, Tóquio. Proceedings. Tóquio, 1997. p.113-114.

SILVA, J. C. P. da; PASCHOARELLI, L. C. Parâmetros antropométricos dos estudantes da cidade de Bauru - SP: da pré-escola à universidade. In: Congresso Braslleiro de Ergonomia, 9., 1999, Salvador. Anais. Rio de J aneiro: ABERG0, 1999. Cd-rom.

TROUSSIER, B.; TESNIERE, C.; FAUCONNIER, J .; GRISON, J .; J UVIN, R.; PHELIP, X. Comparative study of two different kinds of school furniture among children. Ergonomics, London, v. 42, n. 3, p. 516- 526, 1999.

VALENTE, J . A. 0 uso inteligente do computador na educação. Disponível em: <http://www.proinfo.mec.gov.br> Acesso em: 14 maio 2002.

WISNER, A. Por dentro do trabalho: ergonomia: método e técnica. São Paulo: FTD/ Oboré, 1987. 189 p.

Recebido em 12.07 .02

Aprovado em 07.04 .03

Lys Esther Rocha é professora doutora do Departamento de Medicina Legal, Ética Médica e Medicina Social e do Trabalho da Faculdade de Medicina da Universidade de São Paulo.

Raquel Aparecida Casarotto é professora doutora do Departamento de Fisioterapia, Fonoaudiologia e Terapia Ocupacional da Faculdade de Medicina da Universidade de São Paulo.

Laerte Sznelwar é professor doutor do Departamento de Engenharia de Produção da Escola Politécnica da Universidade de São Paulo. 